\title{
Reference distribution of the bioelectrical impedance vector in healthy term newborns
}

\author{
Ana V. B. Margutti ${ }^{1}$, Jacqueline P. Monteiro ${ }^{2}$ and José S. Camelo $\mathrm{Jr}^{2} *$ \\ ${ }^{1}$ University of Ribeirão Preto, São Paulo, Brazil \\ ${ }^{2}$ Department of Pediatrics, School of Medicine of Ribeirão Preto, University of São Paulo, Bandeirantes Avenue, 3900, \\ 7th Floor, Clinics Hospital, Monte Alegre Campus, Ribeirão Preto, São Paulo, Brazil
}

(Received 3 February 2010 - Revised 17 May 2010 - Accepted 24 May 2010 - First published online 2 July 2010)

Bioelectrical impedance vector analysis (BIVA) is a new method that is used for the routine monitoring of the variation in body fluids and nutritional status with assumptions regarding body composition values. The aim of the present study was to determine bivariate tolerance intervals of the whole-body impedance vector and to describe phase angle (PA) values for healthy term newborns aged 7-28d. This descriptive cross-sectional study was conducted on healthy term neonates born at a low-risk public maternity. General and anthropometric neonatal data and bioelectrical impedance data $(800 \mu \mathrm{A}-50 \mathrm{kHz})$ were obtained. Bivariate vector analysis was conducted with the resistance-reactance (RXc) graph method. The BIVA software was used to construct the graphs. The study was conducted on 109 neonates $(52.3 \%$ females) who were born at term, adequate for gestational age, exclusively breast-fed and aged 13 (SD 3.6) d. We constructed one standard, reference, RXc-score graph and RXc-tolerance ellipses (50, 75 and 95\%) that can be used with any analyser. Mean PA was 3.14 (SD 0.43$)^{\circ}(3 \cdot 12 \text { (SD } 0 \cdot 39)^{\circ}$ for males and 3.17 (SD 0.48) $)^{\circ}$ for females). Considering the overlapping of ellipses of males and females with the general distribution, a graph for newborns aged 7-28d with the same reference tolerance ellipse was defined for boys and girls. The results differ from those reported in the literature probably, in part, due to the ethnic differences in body composition. BIVA and PA permit an assessment without the need to know body weight and the prediction error of conventional impedance formulas.

Infants: Newborns: Bioelectrical impedance analysis: Body composition analysis: Impedance vector analysis

Bioelectrical impedance analysis (BIA) is a method that is used for the measurement of ionic electrical conduction of soft tissues, represented by the vector impedance $Z$, which is a combination of resistance $(R)$ and reactance $(\mathrm{Xc})$ through the tissues ${ }^{(1,2)}$.

BIA results are influenced by factors such as the environment, ethnicity, phase of menstrual cycle and underlying medical conditions. It has been suggested that biological and physiological assumptions for the estimation of body composition, which are mainly based on Caucasian samples, may not be accurate for other ethnic groups. There are several factors that are responsible for ethnic differences: fat distribution, body density and differences in proportional limb lengths ${ }^{(3)}$. According to Sluyter et al. ${ }^{(4)}$ who studied healthy adolescents, the relationship between BIA and body composition is ethnicity dependent. Haroun et al. ${ }^{(5)}$ found a significant variability in body composition among different ethnic groups while studying adolescents, which were not reflected by BMI.

The need for predictive BIA equations validated for the population under study can be obviated by using alternative methods such as bioelectrical impedance vector analysis (BIVA) and the study of phase angle (PA $)^{(6)}$. In BIVA, $R$ and Xc corrected for height/length $(H)$ are plotted on the
RXc plane as vector points, and they do not depend on equations or models ${ }^{(6,7)}$. BIVA values are available in the literature for healthy neonates during the first week of life ${ }^{(8)}$, but not during the late neonatal period. PA reflects changes in the electrical conductivity of the body, indicating changes in cell membrane integrity and in the intercellular space ${ }^{(2,8-10)}$.

The hypothesis for the present study was that due to differences in water turnover and the variability in body composition between ethnic groups, the BIVA of neonates aged 7-28d would present vectors differing from those reported in the literature. Thus, the objective of the present study was to establish $R$ - and Xc-corrected values and to construct BIVA curves for healthy 7- to 28-d-old neonates born at term and adequate for gestational age, and also to establish PA values.

\section{Subjects and methods}

This was a descriptive cross-sectional cohort study conducted at a public maternity that attends low complexity cases in Ribeirão Preto, São Paulo, Brazil. Healthy neonates of both sexes aged 7-28d, exclusively breast-fed, and with an adequate weight gain $(25-30 \mathrm{~g} / \mathrm{d})$ and considered to be adequate

Abbreviations: BIA, bioelectrical impedance analysis; BIVA, bioelectrical impedance vector analysis; $H$, height/length; PA, phase angle; $R$, resistance; Xc, reactance. *Corresponding author: Professor J. S. Camelo Jr, fax +55 163602 2700, email jscamelo@fmrp.usp.br 
for gestational age at birth were included in the study. The adequacy of gestational age was determined by the intrauterine growth curve of Alexander et al. ${ }^{(11)}$.

The evaluations were performed during the puerperal return visit $7 \mathrm{~d}$ after delivery. Some inclusion criteria, such as adequate birth weight for gestational age, number of prenatal visits and exclusive breast-feeding, were verified according to the classification of the $\mathrm{WHO}^{(12)}$.

Body weight was measured with the neonate being unclothed and lying on a Filizola Baby ${ }^{\circledR}$ scale, and body length was measured with an Alturexata ${ }^{\circledR}$ horizontal anthropometer.

A monofrequency RJL System ${ }^{\circledR}$ Model Quantum II $(800 \mu \mathrm{A}$ and $50 \mathrm{kHz})$ apparatus was used for measuring bioelectrical impedance. Adhesive electrodes were placed on previously standardised points on the hand and foot. The neonate was positioned in dorsal decubitus, and the bioelectrical impedance data $(R$ and $\mathrm{Xc})$ were obtained when the neonate was still, preferably during calm sleep, avoiding contact between the upper limbs and the trunk, and between the lower limbs. The recommendations of Kyle et $a l .{ }^{(13)}$ were followed, although within the limitations of the neonatal period.

$R$ and $\mathrm{Xc}$ values were measured three times in each infant, and the mean values were used. The apparatus was calibrated after every twenty evaluations using a $500 \Omega$ resistor provided by the manufacturer.

PA was obtained from the arc-tangent ratio $\mathrm{Xc}: R$. To transform the result from radians to degrees, the result that was obtained was multiplied by $180^{\circ} / \pi$ or approximately by $57 \cdot 297^{(14)}$.

The Statistical Analysis Systems $9.1^{\circledR}$ (SAS Institute, Inc., Cary, NC, USA $)^{(15)}$ software was used to calculate the Pearson correlation coefficient $(r)$, and for linear regression, models adjusted with and without the weight variable as a confounder were used. The Hotelling $T^{2}$ test and univariate analysis ( $F$ test) were applied by the BIVA software $2002^{(16)}$ for the analysis of the CI for the comparison of the subject groups and for the analysis of tolerance intervals. The $95 \% \mathrm{CI}$ and the $5 \%$ level of significance were used in all analyses.

The present study was conducted according to the guidelines laid down in the Declaration of Helsinki, and all procedures involving human subjects/patients were approved by the Research Ethics Committee of the University Hospital, School of Medicine of Ribeirão Preto, University of São Paulo. All mothers or persons responsible for the neonates gave written informed consent to participate in the study.

\section{Results}

A total of 109 neonates (fifty-two boys and fifty-seven girls) were evaluated between December 2006 and March 2007.

We determined maternal age, number of pregnancies (including the current one), parity, number of abortions, weeks of gestation when prenatal care was started and number of prenatal visits attended. Data are reported as means and standard deviations. The mean age of the mothers of the neonates studied was 24 (SD 5.3) years. The mean number of pregnancies was 2 (SD 1.5), with a low abortion rate $(0 \cdot 3(\mathrm{SD} 0 \cdot 5))$. The mean number of prenatal visits was 8 (SD 2.0), a value considered adequate by the WHO in situations of prenatal monitoring of low-risk pregnancies ${ }^{(17,18)}$.
The mean gestational age was 39.8 weeks at the time of the study. The birth weight of all the neonates studied was $3297.9 \mathrm{~g}$, and birth length was $0.493 \mathrm{~m}$. The mean neonatal age at the time of the study was $13 \mathrm{~d}$, and the mean body length was $0.507 \mathrm{~m}$. The mean neonatal body weight was greater among boys (3631.7 $\mathrm{g}$ for boys and 3466.6 $\mathrm{g}$ for girls).

Table 1 lists all the values needed for the construction of RXc graphs for BIVA of neonates aged 7-28d, as well as the PA values. These data were also analysed separately based on sex in order to determine whether it would be necessary to construct and later use separate RXc graphs for each sex for this age range. In the first step of the analyses, when only the sex variable was used, associations between sex and $R \quad(P=0.03)$, and between sex and $R / H(P=0.02)$ were detected. No sex association was detected for Xc $(P=0 \cdot 11)$, $\mathrm{Xc} / H(P=0.07)$ or PA $(P=0.59)$. In a second step, when current body weight was added as a possible confounder, it was observed that the association of the sex variable with $R \quad(P=0 \cdot 10)$ and $R / H(P=0 \cdot 13)$ did not persist. The $\mathrm{Xc}$ variable $(P=0.06)$, as well as the $\mathrm{Xc} / H(P=0.07)$ and $\mathrm{PA}$ $(P=0 \cdot 24)$ variables continued to show no association with sex or body weight.

Table 1 shows the anthropometric characteristics and the impedance measurements of the study subjects according to sex, as well as their comparison with the data reported by Piccoli et al. ${ }^{(8)}$, who studied neonates aged $0-7 \mathrm{~d}$, and those reported by Savino et al. ${ }^{(19)}$, who studied infants aged 0-3.99 months.

The values thus obtained permitted the construction of the RXc graphs using the BIVA software $2002^{(16)}$. Fig. 1 presents the impedance vectors with tolerance ellipses of 50, 75 and $95 \%$ for all the neonates, and for boys and girls aged $7-28 \mathrm{~d}$, respectively. The CI shown in Fig. 1 reveal that there is a statistically significant difference between the vectors for girls and boys $(P=0.0382)$ as well as between the remaining vectors $(P=0 \cdot 0000)$, even though the clinical relevance is not defined, considering the correction for weight. Fig. 2 presents the RXc-score graph of impedance vectors with tolerance ellipses of 50, 75 and $95 \%$ for all the neonates, and for boys and girls aged 7-28d, respectively.

\section{Discussion}

In the present study, we obtained the tolerance intervals of the ellipses for BIVA in neonates aged 7-28 d. As a qualitative/ semi-quantitative method for the assessment of hydration and of body tissues, BIVA is clinically useful, and can be used for the routine monitoring of variations in the body fluids and nutritional status of neonates in good condition or in situations requiring special care.

For the estimation of body compartments, the standard BIA method is based on two assumptions, i.e. fixed tissue hydration and the behaviour of the human body as a cylinder that conducts the electrical current homogeneously. If the body water compartments are undergoing strong changes, as is the case for neonates, the calculation is imprecise. In addition, the mean level of fat-free mass hydration varies with age ( $80 \%$ in neonates, $75 \%$ in 10-year-old children and $73 \%$ in healthy adults) ${ }^{(7)}$.

It is known that infancy is characterised by rapid changes and wide interindividual variability in body fluids. Thus, the 
estimates of body composition obtained from BIA during the first few years of life may not be precise since $R$, and consequently the impedance values, may be affected by factors acting on fluid distribution and on free electrolyte concentration between the intra- and extracellular compartments ${ }^{(20)}$. On this basis, during the neonatal period and infancy, the hydration of muscle tissues cannot be considered to be constant ${ }^{(21)}$, and therefore, the standard method for the assessment of body composition by BIA is thought to be inadequate.

In view of the problems encountered with BIA predictive equations, alternative methods using only $R$ and Xc values to assess an individual have been suggested ${ }^{(6)}$. BIVA is a method that does not involve any assumptions about body composition values. Using the RXc graph for healthy neonates, it is possible to monitor the nutritional status and the body fluids of other neonates, with the possibility of predicting a clinical state depending on their location in the quadrants of the graphic ellipses.

Normal body hydration is represented by the positioning of the vector inside the $75 \%$ tolerance ellipsis of sex and age specific for the reference population. Dehydration is represented by an elongated and a steeper curve of the vector, and fluid overload is represented by a reduced and downward inclined vector of the $75 \%$ tolerance ellipsis (outside the interval). Descending vectors or vectors migrating in parallel to the shorter axis above (left) or below (right) the major axis of the tolerance ellipsis, respectively, indicate a smaller or a larger cell mass contained in smooth tissues (vectors with a comparable $R$ value and a higher or lower Xc, respectively) ${ }^{(1,22-24)}$.

According to Piccoli et al. ${ }^{(8)}$, before their publication, no reference value for BIVA of healthy neonates was available in the literature. On comparing our results obtained for healthy neonates during the first week of life, we observed a lower mean $R$ value $(505 \Omega)$ and a higher Xc value $(43 \Omega)$ compared with those reported in the study done by Piccoli et al. ${ }^{(8)}$.

Savino et al. ${ }^{(19)}$ studied 153 infants of both sexes who were divided into three age groups: group A, 0-3.99 months; group B, 4-7.99 months; and group C, 8-11.99 months. They observed that the values obtained for the infants who were evaluated increased progressively with age, except for $R$, which remained similar in the infants of groups $\mathrm{B}$ and C. By comparing these data with those obtained in the present study of healthy neonates aged 7-28 d, it can be observed that the three groups presented lower $R$ and Xc values.

In a study of 115 healthy infants born at term and aged less than 6 months, Savino et al. ${ }^{(25)}$ detected lower $R$ and $\mathrm{Xc}$ values than those detected in the present study, in which only neonates were evaluated.

The use of BIVA has proved to be clinically useful in preschoolers, schoolchildren, adolescents and adults under body fluid monitoring ${ }^{(2,6,26)}$, and to discriminate between obese individuals and individuals with oedema ${ }^{(27)}$.

Since the water turnover of neonates is considerable, the clinical applicability of these reference values of BIVA will have to be tested later in neonates aged 7-28d, especially regarding the monitoring of the water balance and in situations in which changes in extracellular volume are observed without a change in total body volume, when weight is not a reliable parameter $^{(28-31)}$.

Another alternative to standard BIA is the use of PA. Studies have suggested that PA can be useful to determine 

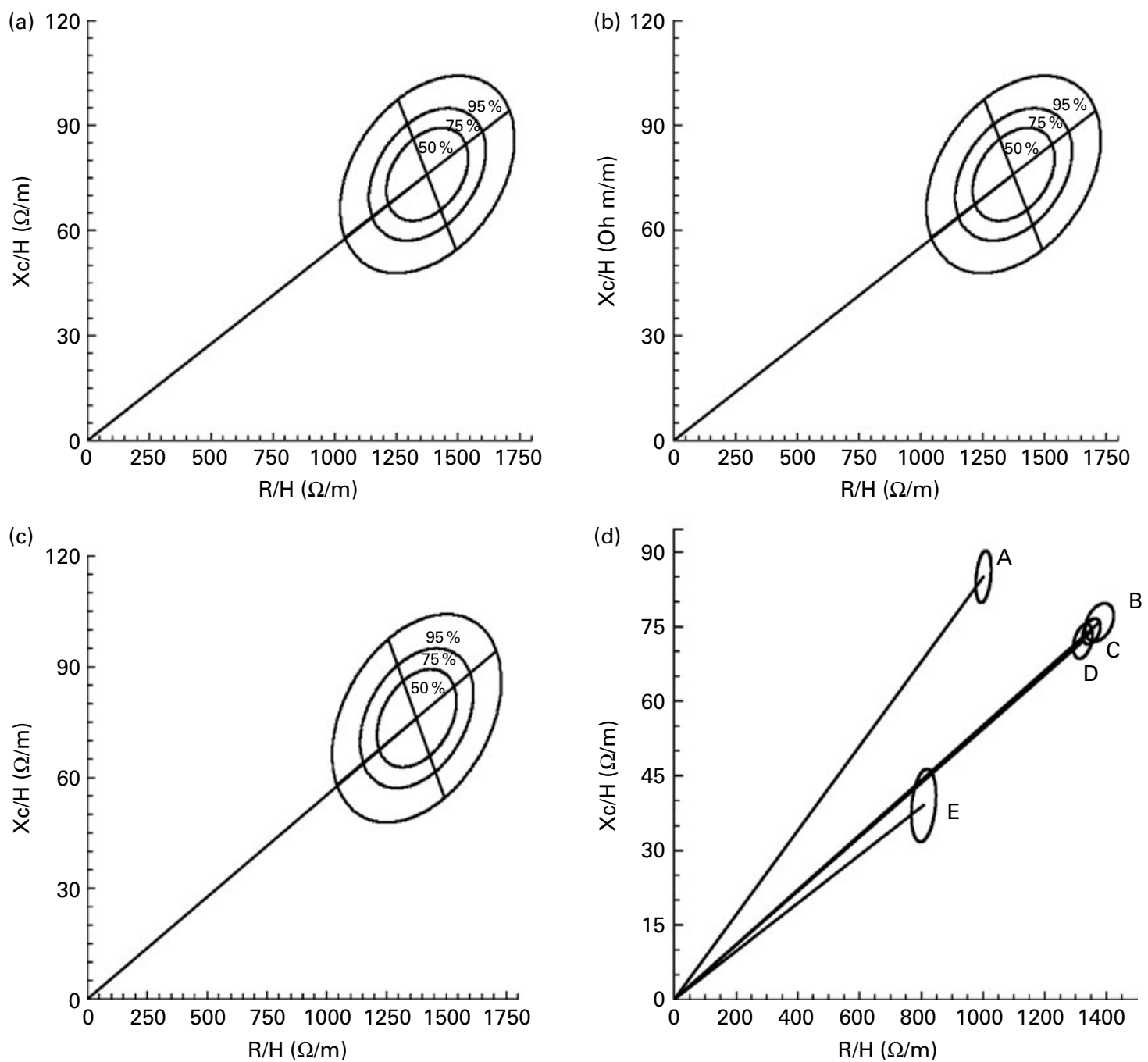

Fig. 1. Graphs of the impedance vector with the 50,75 and $95 \%$ tolerance ellipses for (a) all the neonates, (b) male neonates and (c) female neonates aged 7-28d, and (d) impedance vectors with $95 \%$ confidence ellipses for healthy children (Hotelling $T^{2}$ test). Comparison graph shown for (A) study done by Piccoli et al. $\left.{ }^{(8)}\right)$ - all neonates $(0-7 \mathrm{~d})$; (B) present study - female neonates (7-28d); (C) present study - all neonates (7-28d); (D) present study - male neonates (7-28 d); (E) study done by Savino et al. ${ }^{(19)}$ - all newborns and young infants $(0-3.99$ months). $R / H$, resistance/length; $\mathrm{Xc} / H$, reactance/length.

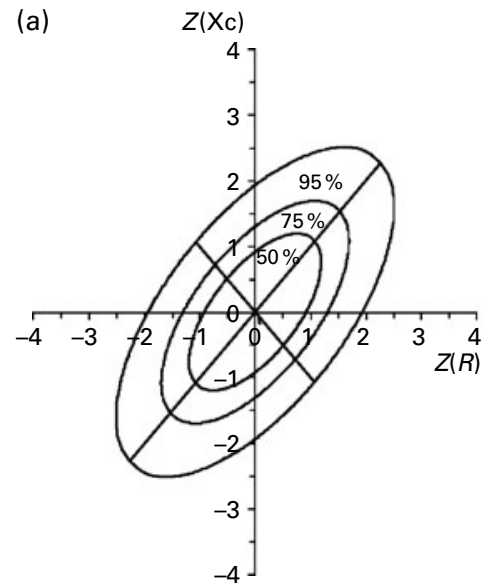

(b)

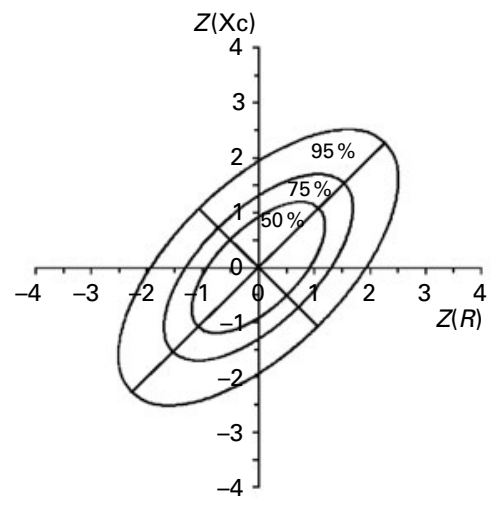

(c)

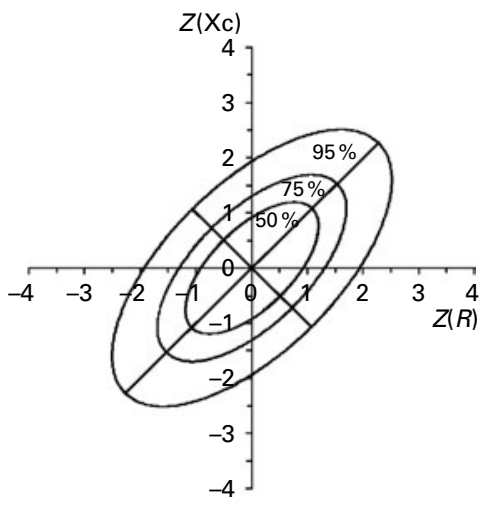

Fig. 2. Resistance-reactance-score graph of impedance vector with the 50, 75 and $95 \%$ tolerance ellipses for (a) all the neonates, (b) male neonates and (c) female neonates aged $7-28 \mathrm{~d}$. $R / H$, resistance/length; $\mathrm{Xc} / H$, reactance/length. 
an increased risk of morbidity, with lower PA values being relevant to the prognosis ${ }^{(7,13)}$. Lower PA values may be associated with cell death or with some change in selective membrane permeability. Higher values may be associated with a greater quantity of intact cell membranes, i.e. a greater body cell mass ${ }^{(13,32-34)}$. The PA values of the infants studied by Savino et al. ${ }^{(25)}$ were higher than the present ones regardless of the type of feeding. Compared with the PA values obtained in the study done by Savino et al. ${ }^{(19)}$ on infants aged 0-3.99 months and 4-7.99 months, the present PA values were higher. Piccoli et al. ${ }^{(8)}$ reported higher PA values than obtained here.

On this basis, we conclude that for the BIVA of newborns aged 7-28d, specific values should be used since they differ from the values of newborns in the first week of life, from those for young infants and from the remaining data reported in the literature. The difference with regard to other populations might be explained in part by different ethnicity. Although the Hotelling $T^{2}$ test showed a significant difference between boys and girls, the significance of the correlations between BIVA parameters and sex, which are not consistent correcting for weight could not be clinically relevant, is not clear. In addition, an overlapping of ellipses of males and females with the general distribution is visible. Therefore, the general distribution should be used for boys and girls.

\section{Acknowledgements}

The authors wish to thank Professor Antonio Piccoli, University of Padua, Italy, for kindly providing the BIVA Software 2002 (Piccoli \& Pastori ${ }^{(16)}$; available from apiccoli@unipd.it) and Davi Casale Aragon for statistical assistance. The authors disclose that there are no conflicts of interests in the present paper. A. V. B. M., J. P. M. and J. S. C. participated in the data collection, statistical analysis and writing of the manuscript. The present study was supported by an MSc grant to A. V. B. M. (CAPES, Brazil).

\section{References}

1. Kushner RF (1992) Bioelectrical impedance analysis: a review of principles and applications. J Am Coll Nutr 11, 199-209.

2. De Palo T, Messina G, Edefonti A, et al. (2000) Normal values of the bioelectrical impedance vector in childhood and puberty. Nutrition 16, 417-424.

3. Dehghan M \& Merchant AT (2008) Is bioelectrical impedance accurate for use in large epidemiological studies? Nutr J 7, 26.

4. Sluyter JD, Schaaf D, Scragg RKR, et al. (2009) Prediction of fatness by standing 8-electrode bioimpedance: a multiethnic adolescent population. Obesity (Silver Spring) 18, 183-189.

5. Haroun D, Taylor SJ, Viner RM, et al. (2009) Validation of bioelectrical impedance analysis in adolescents across different ethnic groups. Obesity (Silver Spring) (Epublication ahead of print version 29 October 2009).

6. Piccoli A, Rossi B, Pillon L, et al. (1994) A new method for monitoring body fluid variation by bioimpedance analysis: the RXc graph. Kidney Int 46, 534-539.

7. Kyle UG, Bosaeus I, De Lorenzo A, et al. (2004) Bioelectrical impedance analysis - part I: review of principles and methods. Clin Nutr 23, 1226-1243.
8. Piccoli A, Fanos V, Peruzzi L, et al. (2002) Reference values of the bioelectrical impedance vector in neonates in the first week after birth. Nutrition 18, 383-387.

9. Scheltinga MR, Jacobs DO, Kimbrough TD, et al. (1991) Alterations in body fluid can be detected by bioelectrical impedance analysis. J Surg Res 50, 461-468.

10. Pupim LCB, Ribeiro CB, Kent P, et al. (2000) Atualização em diálise: Uso da impedância bioelétrica em pacientes em diálise (Update on dialysis: use of bioelectrical impedance in dialysis patients). J Bras Nefrol 22, 249-256.

11. Alexander GR, Himes JH, Kaufman RB, et al. (1996) A United States national reference for fetal growth. Obst Gynecol 87, $163-168$.

12. World Health Organization (1992) Indicadores para avaliar as prácticas de lactância materna (Indicators for Assessing the Practices of Maternal Lactation). Geneva: WHO.

13. Kyle UG, Bosaeus I, De Lorenzo A, et al. (2004) Bioelectrical impedance analysis - part II: review of principles and methods. Clin Nutr 23, 1430-1453.

14. Baumgartner RN, Chumlea WC \& Roche PA (1988) Bioelectric impedance phase angle and body composition. Am J Clin Nutr 48, 16-23.

15. SAS Institute, Inc. (2002) SAS/STAT ${ }^{\circledR}$ User's Guide, version 9. Cary, NC: SAS Institute, Inc.

16. Piccoli A \& Pastori G (2002) BIVA software. Padova: Department of Medical and Surgical Sciences, University of Padova.

17. Ribeiro ERO, Guimarães AMDN, Bettiol H, et al. (2009) Risk factors for inadequate prenatal care use in the metropolitan area of Aracaju, Northeast Brazil. BMC Pregnancy Childbirth 9, $31-38$.

18. Carroli G, Villar J, Piaggio G, et al. (2001) WHO systematic review of randomized controlled trials of routine antenatal care. Lancet 357, 1565-1570.

19. Savino F, Grasso G, Cresi F, et al. (2003) Bioelectrical impedance vector distribution in the first year of life. Nutrition 19, 492-496.

20. Baumgartner RN (1996) Electrical impedance and total body electrical conductivity. In Human Body Composition, pp. 79-102 [PA Roche, SB Heymsfield and TG Lohman, editors]. Champaign, IL: Human Kinetics.

21. Barbosa-Silva MCG, Barros AJD, Wang J, et al. (2005) Bioelectrical impedance analysis: population reference values for phase angle by age and sex. Am J Clin Nutr 82, 49-52.

22. Foster KF \& Lukaski HC (1996) Whole-body impedance what does it measure? Am J Clin Nutr 64, 388S-396S.

23. Heymsfield SB, Wang ZM, Visser M, et al. (1996) Techniques used in the measurement of body composition: an overview with emphasis on bioelectrical impedance analysis. Am J Clin Nutr 64, 478S-484S.

24. Kushner RF, Schoeller DA, Fjeld CR, et al. (1992) Is the impedance index $\left(\mathrm{ht}^{2} / \mathrm{R}\right)$ significant in predicting total body water? Am J Clin Nutr 56, 835-839.

25. Savino F, Liguori SA, Fissore MF, et al. (2008) Looking for a relation between serum leptin concentration and body composition parameters in healthy term infants in the first 6 months of life. J Pediatr Gastroenterol Nutr 46, 348-351.

26. Piccoli A (1998) Identification of operational clues to dry weight prescription in hemodialysis using bioimpedance vector analysis. Kidney Int 53, 1036-1043.

27. Piccoli A, Brunani A, Salvia G, et al. (1998) Discriminating between body fat and fluid changes in the obese adults using bioimpedance vector analysis. Int $J$ Obes Relat Metab Disord 22, 97-104.

28. El-Dahr SS \& Chevalier RL (1990) Special needs of the newborn infant in fluid therapy. Pediatr Clin North Am 37, $323-336$. 
29. Tang W, Ridout D \& Modi N (1997) Assessment of total body water using bioelectrical impedance analysis in neonates receiving intensive care. Arch Dis Child 77, F123-F126.

30. Ferreira DM \& Souza MN (2004) Bioelectrical impedance spectroscopy for the assessment of body fluid volumes of term neonates. Braz J Med Biol Res 37, 1595-1606.

31. Dung NQ, Fusch G, Armbrust S, et al. (2007) Body composition of preterm infants measured during the first months of life: bioelectrical impedance provides insignificant additional information compared to anthropometry alone. Eur J Pediatr 166, $215-222$.
32. Peters BSE, Jorgetti V \& Martini LA (2006) Body composition changes in hemodialysis patients with secondary hyperparathyroidism after parathyroidectomy measured by conventional and vector bioimpedance analysis. Br J Nutr $\mathbf{9 5}$, 253-257.

33. Nagano M, Suita S \& Yamanouchi T (2000) The validity of bioelectrical impedance phase angle for nutritional assessment in children. J Pediatr Surg 35, 1035-1039.

34. Azevedo ZMA, Silva DR, Dutra MVP, et al. (2007) Associação entre Ângulo de Fase, PRISM I e Gravidade da Sepse (Association between phase angle, PRISM I and severity of sepsis). Rev Bras Terapia Int 19, 297-303. 